\title{
Motive für freiwilliges Engagement, Beendigungsgründe, Hinderungsgründe und Engagementbereitschaft
}

\author{
Céline Arriagada \& Nora Karnick
}

\section{Kernaussagen}

Als Motive für freiwilliges Engagement werden am häufigsten Spaß und Hilfe für andere Menschen genannt. 93,9 Prozent der Engagierten geben Spaß als Motiv für ihr freiwilliges Engagement an. Anderen Menschen zu helfen wird von 88,5 Prozent der Befragten als Motiv genannt. Am seltensten wird angegeben, an Ansehen und Einfluss gewinnen (26,4 Prozent) oder etwas dazuverdienen (6,1 Prozent) zu wollen.

Engagierte im Alter ab 65 Jahren üben ihr Engagement anteilig besonders häufig aus, um mit anderen Menschen zusammenzukommen. Die 14- bis 29-Jährigen dagegen nennen anteilig besonders häufig das Motiv, eine Qualifikation erwerben zu wollen. Unterschiede zwischen den Geschlechtern sind im Gegensatz zu altersspezifischen Unterschieden nur gering.

Die am häufigsten genannten Beendigungsgründe für freiwilliges Engagement sind berufliche Gründe gefolgt von der zeitlichen Begrenzung der Tätigkeit. Bei 43,3 Prozent der früher Engagierten führen berufliche Gründe zur Beendigung des Engagements, während 42,9 Prozent die zeitliche Begrenzung der Tätigkeit als Grund für die Beendigung nennen. Die am seltensten genannten Beendigungsgründe sind hingegen fehlende Anerkennung (8,o Prozent) oder Schwierigkeiten innerhalb der Gruppe (7,7 Prozent).

Frauen beenden ihr Engagement anteilig häufiger aus familiären Gründen als Männer. Männer hingegen beenden ihr Engagement anteilig am häufigsten aus beruflichen Gründen. Früher Engagierte im Alter von 65 Jahren und älter beenden ihr Engagement häufig, weil sie keine Verpflichtung mehr haben möchten, sie eine Altersgrenze erreicht haben oder aus gesundheitlichen Gründen. 
Der mit Abstand am häufigsten genannte Hinderungsgrund, ein freiwilliges Engagement aufzunehmen, ist fehlende Zeit. 71,3 Prozent der nie engagierten Personen geben zeitliche Gründe an. Dieser Grund wurde von Frauen und Männern sowie über alle Altersgruppen hinweg am häufigsten genannt.

Personen ab 65 Jahre geben anteilig häufiger als jüngere Personen als Grund für die Nichtaufnahme eines Engagements gesundheitliche Gründe oder den Wunsch, keine Verpflichtung eingehen zu wollen, an. Personen im Alter zwischen 30 und 64 Jahren geben anteilig häufiger als die Personen der jüngsten und der höchsten Altersgruppe berufliche Gründe für die Nichtaufnahme eines Engagements an. Frauen geben familiäre Gründe anteilig häufiger an, während Männer anteilig häufiger berufliche Gründe nennen.

Die Mehrheit der aktuell Nicht-Engagierten kann sich vorstellen, in Zukunft eine freiwillige Tätigkeit aufzunehmen. 58,7 Prozent der aktuell nicht-engagierten Befragten haben dies angegeben. Die Bereitschaft ein freiwilliges Engagement zu beginnen ist bei Menschen zwischen 14 und 29 Jahren besonders hoch. Insgesamt 82,4 Prozent der Nicht-Engagierten in der jüngsten Altersgruppe können sich prinzipiell vorstellen, eine freiwillige Tätigkeit aufzunehmen. Bei den Nicht-Engagierten ab 65 Jahren sind dies mit 27,2 Prozent deutlich weniger. 


\subsection{Einleitung}

Menschen finden auf unterschiedlichen Wegen ins Engagement. Häufig engagieren sie sich aus Eigeninitiative, doch ebenso häufig ergeben sich Anstöße aus dem persönlichen Umfeld, etwa durch die Familie, durch den Freundeskreis oder über Bekannte (Müller et al. 2017). Weiterhin gibt es eine Reihe verschiedener Kampagnen und Projekte, die unterschiedliche Zielgruppen zur Aufnahme eines Engagements motivieren wollen oder bereits Engagierte unterstützen. Das Projekt „Engagierte Stadt" (Engagierte Stadt 2020) beispielsweise soll die Zusammenarbeit und Abstimmung zwischen den verschiedenen teilnehmenden Organisationen und Vereinen vor Ort fördern. Das Bundesprogramm „Mehrgenerationenhaus“ (Bundesministerium für Familie, Senioren, Frauen und Jugend (BMFSFJ) 2020a) fördert bundesweit über 540 Mehrgenerationenhäuser, die sich explizit an Menschen verschiedener Generationen, aber auch unterschiedlicher Herkunft oder Konfession richten. Gut organisierte und vernetzte Projekte und Organisationen können den Zugang zu freiwilligem Engagement für Interessierte erheblich erleichtern. Andere Kampagnen wie „Menschen stärken Menschen“ (BMFSFJ 2020b), „Deine Zeit ist jetzt“ (Bundesanstalt Technisches Hilfswerk (THW) 2020) oder „Mein Ehrenamt - Mein Moment“ (Hessische Landesregierung 2020) richten sich direkt an freiwillig Engagierte oder jene, die sich vorstellen können in Zukunft eine freiwillige Tätigkeit zu beginnen. Dabei werden, hauptsächlich online, auf verschiedenen Plattformen Informationen zu freiwilligem Engagement angeboten, Engagierte geehrt oder auch Ehrenamtssuchmaschinen zur Verfügung gestellt, um Interessierte zu erreichen und den Zugang zu freiwilligem Engagement zu erleichtern.

Engagierte haben bestimmte subjektive Erwartungen an ihre Tätigkeit, die sie zu ihrem Engagement motivieren (Shye 2009; Wenzel et al. 2012). Die Strukturen, die die Möglichkeiten für freiwilliges Engagement fördern, müssen daher auf entsprechende Motive treffen, damit Menschen sich engagieren (Institut für Demoskopie Allensbach 2013). Diese Motive können unterschiedlicher Natur sein. Die Freude am freiwilligen Engagement ist dabei der stärkste Antrieb und steht bei den meisten Engagierten im Vordergrund (Haumann 2014; Institut für Demoskopie Allensbach 2013; Müller et al. 2017). Zudem scheinen vor allem jene Motive weit verbreitet, welche die sozialen Funktionen des Engagements betreffen (Shye 2009). So engagieren sich Personen, um mit anderen Menschen zusammenzukommen, ihnen zu helfen und die Gesellschaft mitzugestalten. Stärker materiell geprägte und auf den eigenen Nutzen ausgerichtete Motive, wie der Erwerb von Qualifikationen oder der Zuverdienst durch die freiwillige Tätigkeit, sind dagegen eher nachgeordnet (Institut für Demoskopie Allensbach 2013; Müller et al. 2017; Seebauer 2013). Die aktive Entscheidung zum freiwilligen Engagement ist häufig Ausdruck eines „komplexen Motivbündel[s]“ (Haumann 2014: 5). Das freiwillige Engagement fußt dabei sowohl auf altruistischen Motiven und auf dem Nutzen für die Gemeinschaft als auch auf eigennützigen Motiven, wie dem individuellen Mehrwert und der „aktive[n] Selbst- 
gestaltung der eigenen Biografie" (Seebauer 2013: 7; Haumann 2014; Moschner 2002; Wagner 2019).

Im Freiwilligensurvey 2019 werden aktuell engagierte Personen zu neun unterschiedlichen Motiven für ihr freiwilliges Engagement befragt. Wir unterscheiden zwischen altruistischen Motiven (anderen Menschen helfen, etwas für das Gemeinwohl tun, die Gesellschaft mitgestalten sowie Gutes zurückgeben, weil selbst Engagement erfahren wurde), sozial geprägten Motiven (Spaß haben und mit Menschen zusammenkommen) sowie Motiven eigennütziger Natur (Qualifikationen erwerben, Ansehen und Einfluss gewinnen sowie etwas dazuverdienen). Zur Ergänzung der Motivabfrage wurden 2019 drei Motive zu gemeinnützigen Aspekten zusätzlich aufgenommen: so etwa der Wunsch, etwas für das Gemeinwohl zu tun sowie das Motiv, anderen zu helfen. Ein weiteres neu aufgenommenes Motiv zielt auf einen Aspekt der Reziprozität des freiwilligen Engagements ab, und zwar der Wunsch, etwas zurückzugeben, weil einem selbst Gutes durch das Engagement anderer Menschen widerfahren ist (Institut für Demoskopie Allensbach 2013; Moschner 2002; Wagner 2019).

Engagementbiografien können durch vorübergehende Unterbrechungen oder die Beendigung des Engagements gekennzeichnet sein. Diese Unterbrechungen können dabei mehrfach auftreten und sind häufig an bestimmte Lebensphasen geknüpft (Haumann 2014). Engagierte brauchen also nicht nur einen Antrieb, um ein Engagement aufzunehmen, sondern auch, um es dauerhaft auszuüben. Bleibt die Erfüllung des Motivs aus oder werden die Erwartungen an das Engagement enttäuscht, können Engagierte ihre Tätigkeit beenden (Wenzel et al. 2012). Für die Beendigung des Engagements kann es neben Gründen, die in der freiwilligen Tätigkeit selbst liegen, auch Gründe außerhalb des Engagements geben, beispielsweise persönliche Lebensumstände (Haumann 2014; Wenzel et al. 2012). Die Vereinbarkeit von Beruf, Familie und Engagement scheint dabei eine große Rolle zu spielen: Engagierte im mittleren Alter sehen sich einer vielfachen Belastung ausgesetzt, die sich durch berufliche und familiäre Aufgaben ergibt. (Haumann 2014; Institut für Demoskopie Allensbach 2013; Müller \& Tesch-Römer 2017). In den höheren Altersgruppen wird insbesondere aufgrund gesundheitlicher Faktoren und Altersgrenzen entschieden, ob eine freiwillige Tätigkeit ausgeübt wird oder nicht (Müller \& Tesch-Römer 2017; Wenzel et al. 2012). Im Freiwilligensurvey 2019 werden ehemals engagierte Personen gefragt, welche privaten oder tätigkeitsbezogenen Gründe zur Beendigung ihres Engagements geführt haben. Mit dem Gefühl fehlender Anerkennung wurde 2019 ein weiterer Beendigungsgrund aufgenommen. Die Mehrheit der Engagierten fühlt sich für ihre Arbeit wertgeschätzt (Institut für Demoskopie Allensbach 2013). Fehlt diese Anerkennung allerdings, kann das zu Enttäuschungen führen und freiwillig Engagierte dazu bewegen, ihr Engagement zu beenden.

Die Gründe, aus denen früher engagierte Personen ihr Engagement beendet haben, decken sich größtenteils mit den Gründen, die bisher nie engagierte Personen als Hinderungsgründe, ein Engagement aufzunehmen, nennen. Auch hier spielen zeitliche, berufliche und familiäre Belastungen eine große Rolle oder auch der 
Wunsch, keine Verpflichtungen einzugehen (Müller \& Tesch-Römer 2017; Seebauer 2013; Wenzel et al. 2012). Dennoch ist die Bereitschaft zum freiwilligen Engagement als hoch einzuschätzen: Bisherige Befunde zeigen, dass mehr als die Hälfte Nicht-Engagierter sich ein zukünftiges Engagement vorstellen könnte (Müller \& Tesch-Römer 2017).

In diesem Kapitel nehmen wir drei Personengruppen mit unterschiedlicher Engagementerfahrung in den Blick: Personen, die aktuell freiwillig engagiert sind, werden nach ihren Motiven befragt. Personen, die früher freiwillig engagiert waren und ihre freiwillige Tätigkeit aufgegeben haben, werden nach den Beendigungsgründen befragt. Schließlich werden Personen, die noch nie freiwillig engagiert waren, nach Hinderungsgründen gefragt. Zusätzlich wird die Engagementbereitschaft aktuell Nicht-Engagierter erfragt. Das Kapitel behandelt folgende Fragen:

a) Welche Motive geben engagierte Personen für ihr freiwilliges Engagement an? Wie unterscheiden sich die Motive nach Geschlecht und nach Altersgruppen?

b) Welche Beendigungsgründe geben Personen an, die sich früher engagiert haben? Wie unterscheiden sich die Beendigungsgründe nach Geschlecht und nach Altersgruppen?

c) Welche Hinderungsgründe geben Personen an, die bisher kein freiwilliges Engagement ausüben? Wie unterscheiden sich die Hinderungsgründe nach Geschlecht und nach Altersgruppen?

d) Wie hoch ist die Engagementbereitschaft von Personen, die derzeit nicht engagiert sind? Wie unterscheiden sich die Anteile von Personen, die bereit sind zukünftig ein Engagement zu übernehmen, nach Geschlecht und nach Altersgruppen?

\subsection{Daten und Indikatoren}

Die Ergebnisse des vorliegenden Kapitels beruhen auf den Daten des Deutschen Freiwilligensurveys (FWS) und werden gewichtet dargestellt. Die Gewichtung zielt darauf ab, die Repräsentativität zu verbessern, um verallgemeinerbare Aussagen treffen zu können. In den Veröffentlichungen zu den Erhebungen der Jahre 1999 bis 2014 wurden im Rahmen des Freiwilligensurveys bislang die Gewichtungsmerkmale Geschlecht, Alter, Bundesland sowie Gemeindegrößenklasse herangezogen. Diese Gewichtung wird nun um das Gewichtungsmerkmal der schulischen Bildung ergänzt. Personen aus unterschiedlichen Bildungsgruppen nehmen mit unterschiedlicher Wahrscheinlichkeit an Umfragen teil; daher sind verschiedene Bildungsgruppen in den Daten über- beziehungsweise unterrepräsentiert. Die Gewichtung inklusive schulischer Bildung wird in diesem Bericht auf die Daten aller fünf Erhebungswellen von 1999 bis 2019 angewendet. Die Engagementquoten fallen durch dieses Vorgehen für alle Erhebungswellen niedriger aus als bisher berichtet; es ändert sich somit das 
Niveau, der Trend bleibt jedoch im Wesentlichen bestehen. Die Gewichtung der Daten inklusive Bildung wirkt sich auch auf andere Ergebnisse aus.

Alle Ergebnisse werden auf statistische Signifikanz getestet (verwendet wird ein Signifikanzniveau von $\mathrm{p}<0,05)$. Die Prüfung auf statistische Signifikanz zeigt an, ob ein anhand der Daten des Freiwilligensurveys gefundener Unterschied mit großer Wahrscheinlichkeit auch in der Grundgesamtheit, also beispielsweise in der Wohnbevölkerung Deutschlands, vorhanden ist (statistisch signifikant) oder nur zufällig in den Daten vorliegt (statistisch nicht signifikant).

Die dargestellten Ergebnisse fußen auf der Befragung des Freiwilligensurveys 2019, die von März bis November 2019 erfolgte. Somit ist es mithilfe der Daten des Freiwilligensurveys 2019 nicht möglich, Aussagen über spätere Entwicklungen wie beispielsweise die Auswirkungen der Corona-Pandemie auf das freiwillige Engagement zu tätigen.

Die Ergebnisse werden auf Basis verschiedener Gruppen berichtet. Diese Gruppen sind in Abbildung 6-1 dargestellt. Jede Gruppe repräsentiert einen aktuellen Engagementstatus: (a) 39,7 Prozent der befragten Personen, sind aktuell engagiert; (b) 22,3 Prozent der Personen waren früher engagiert und haben ihr freiwilliges Engagement beendet; und (c) 37,0 Prozent der befragten Personen waren noch nie engagiert. Weiterhin gibt es eine kleine Gruppe (1,o Prozent), zu der keine vollständige Information vorliegt, ob sie aktuell engagiert sind oder in der Vergangenheit engagiert waren.

In diesem Kapitel werden vier unterschiedliche Aspekte betrachtet: die Motive für freiwilliges Engagement, die Beendigungs- sowie Hinderungsgründe für freiwilliges Engagement und die Bereitschaft, sich in Zukunft zu engagieren.

Motive für freiwilliges Engagement: Im Deutschen Freiwilligensurvey 2019 wird erfasst, welche Motive aktuell freiwillig Engagierte (a) für ihr Engagement angeben. Bei der Abfrage der Motive sind Mehrfachnennungen möglich.

Abbildung 6-1 Engagementstatus in der Bevölkerung Deutschlands ab 14 Jahren 2019 (Basis: alle Befragten)

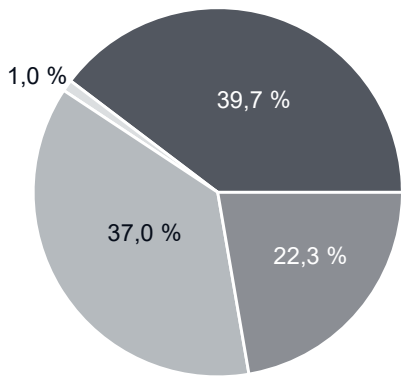

Aktuell engagierte Personen Früher engagierte Personen Nie engagierte Personen Keine Information 
Sagen Sie mir bitte, ob Sie den folgenden Aussagen über Ihr Engagement voll und ganz zustimmen, eher zustimmen, teils/teils zustimmen, eher nicht zustimmen oder ganz und gar nicht zustimmen.

(1) Ich will die Gesellschaft zumindest im Kleinen mitgestalten.

(2) Ich will vor allem mit anderen Menschen zusammenkommen.

(3) Ich will Ansehen und Einfluss in meinem Lebensumfeld gewinnen.

(4) Ich will Qualifikationen erwerben, die im Leben wichtig sind.

(5) Ich will mir durch das Engagement etwas dazuverdienen.

(6) Mein Engagement macht mir Spaß.

(7) Ich will etwas für das Gemeinwohl tun.

(8) Ich will Gutes zurückgeben, weil ich selbst Engagement von anderen erfahren habe.

(9) Ich will damit anderen Menschen helfen.

Mehrfachnennungen möglich; Antwortkategorien (jeweils): Stimme voll und ganz zu; Stimme eher zu; Teils/teils; Stimme eher nicht zu; Stimme ganz und gar nicht zu

Für die Analysen wurden die Kategorien ,Stimme voll und ganz zu' und ,Stimme eher zu' zusammengefasst.

Beendigungsgründe: Weiterhin werden alle Personen, die früher engagiert waren, aber aktuell nicht mehr engagiert sind (b) nach den Gründen für die Beendigung ihres Engagements befragt. Bei der Abfrage der Beendigungsgründe sind Mehrfachnennungen möglich.

Warum haben Sie Ihr Engagement damals beendet?

(1) Der zeitliche Aufwand war zu groß.

(2) Aus gesundheitlichen Gründen.

(3) Aus familiären Gründen.

(4) Aus beruflichen Gründen.

(5) Es gab Schwierigkeiten innerhalb der Gruppe.

(6) Die Tätigkeit war zeitlich begrenzt.

(7) Ich wollte keine Verpflichtungen mehr haben.

(8) Es gab eine Altersgrenze, die ich erreicht habe.

(9) Mir fehlte die Anerkennung.

Mehrfachnennungen möglich; Antwortkategorien (jeweils): Ja, trifft zu; Nein, trifft nicht $z u$

Die Ergebnisse werden je Beendigungsgrund für den Anteil ,Ja, trifft zu' dargestellt.

Hinderungsgründe: Die Frage zu den Hinderungsgründen wird allen Personen gestellt, die noch nie engagiert waren (c). Bei der Abfrage der Hinderungsgründe sind Mehrfachnennungen möglich. 
Es gibt ja viele Gründe, warum man kein freiwilliges Engagement ausübt. Ich lese Ihnen nun einige Gründe vor, sagen Sie mir bitte jeweils, ob dieser Grund auf Sie zutrifft oder nicht. Ist das bei Ihnen ...

(1) Aus zeitlichen Gründen?

(2) Aus gesundheitlichen Gründen?

(3) Aus familiären Gründen?

(4) Aus beruflichen Gründen?

(5) Weil Sie sich nicht dafür geeignet fühlen?

(6) Weil andere, zum Beispiel die Organisation oder Gruppe, Sie abgelehnt haben?

(7) Weil Sie keine Verpflichtungen eingehen wollen?

(8) Weil Sie nicht wissen, wohin Sie sich dafür wenden können?

Mehrfachnennungen möglich; Antwortkategorien (jeweils): Ja, trifft zu; Nein, trifft nicht $z u$

Die Ergebnisse werden je Hinderungsgrund für den Anteil ,Ja, trifft zu'berichtet.

Engagementbereitschaft: Allen derzeit nicht-engagierten Personen, also denjenigen, die ihr Engagement beendet haben (b) oder nie engagiert waren (c), wurde die folgende Frage gestellt:

Wären Sie bereit, sich zukünftig zu engagieren und freiwillig oder ehrenamtlich Aufgaben zu übernehmen? Würden Sie sagen: „Ja, sicher“, „ja, vielleicht“ oder „nein“?

Antwortkategorien: Ja, sicher; Ja, vielleicht; Nein

Diese Frage wurde im Jahr 2014 im Vergleich zu den Wellen 1999, 2004 und 2009 modifiziert, um die zuvor verwendete Formulierung zu verbessern und sie kürzer, klarer und in einem Telefoninterview leichter verständlich zu machen. In den Wellen von 1999, 2004 und 2009 lautete die Formulierung:

Wären Sie heute oder zukünftig interessiert, sich in Vereinen, Initiativen, Projekten oder Selbsthilfegruppen zu engagieren und dort Aufgaben oder Arbeiten zu übernehmen, die man freiwillig oder ehrenamtlich ausübt?

Auch die Antwortmöglichkeiten der Vorwellen ,ja', ,vielleicht, kommt drauf an' und ,nein' unterscheiden sich zum Vorgehen in den Jahren 2014 und 2019.

Aufgrund der Veränderung der Abfrage müssen Vergleiche in der Engagementbereitschaft zwischen den Erhebungsjahren 1999, 2004 und 2009 und den Jahren 2014 und 2019 mit Vorsicht betrachtet werden, da die gestellte Frage über die Erhebungswellen hinweg nicht identisch war. Inhaltlich gleiche Fragen können aufgrund unterschiedlicher Formulierungen von den Befragten unterschiedlich verstanden werden.

Für die Analysen wurden die Kategorien ,Ja, sicher' und ,Ja, vielleicht' beziehungsweise ,Ja' und ,Vielleicht, kommt drauf an' zusammengefasst. 
Die Ergebnisse zu Motiven, Beendigungs- und Hinderungsgründen sowie zur Engagementbereitschaft sind für die in Deutschland lebende Bevölkerung ab 14 Jahren sowie nach Geschlecht und nach Altersgruppen dargestellt. Die verwendeten Differenzierungsmerkmale werden in Kapitel 2 beschrieben.

\subsection{Motive für Engagement}

Das anteilig am häufigsten genannte Motiv der freiwillig Engagierten für ihr Engagement ist der Spaß, den sie bei der Ausübung empfinden. Insgesamt 93,9 Prozent der Engagierten stimmen der Aussage zu, dass ihnen ihr Engagement Spaß bereitet (Abbildung 6-2). Altruistische Aspekte stellen weitere wichtige Motive dar. Die Möglichkeiten anderen Menschen zu helfen (88,5 Prozent) und etwas für das Gemeinwohl zu tun (87,5 Prozent) sind anteilig häufige Beweggründe für freiwilliges Engagement. Von den Engagierten geben 80,4 Prozent an, dass sie ihr Engagement ausüben, um dadurch die Gesellschaft zumindest im Kleinen mitzugestalten. Der soziale Aspekt - nämlich die Gelegenheit mit Menschen zusammen zu kommen -

Abbildung 6-2 Angaben der freiwillig Engagierten zu den Motiven für ihr Engagement 2019 (Basis: alle Engagierten; Mehrfachnennungen möglich)

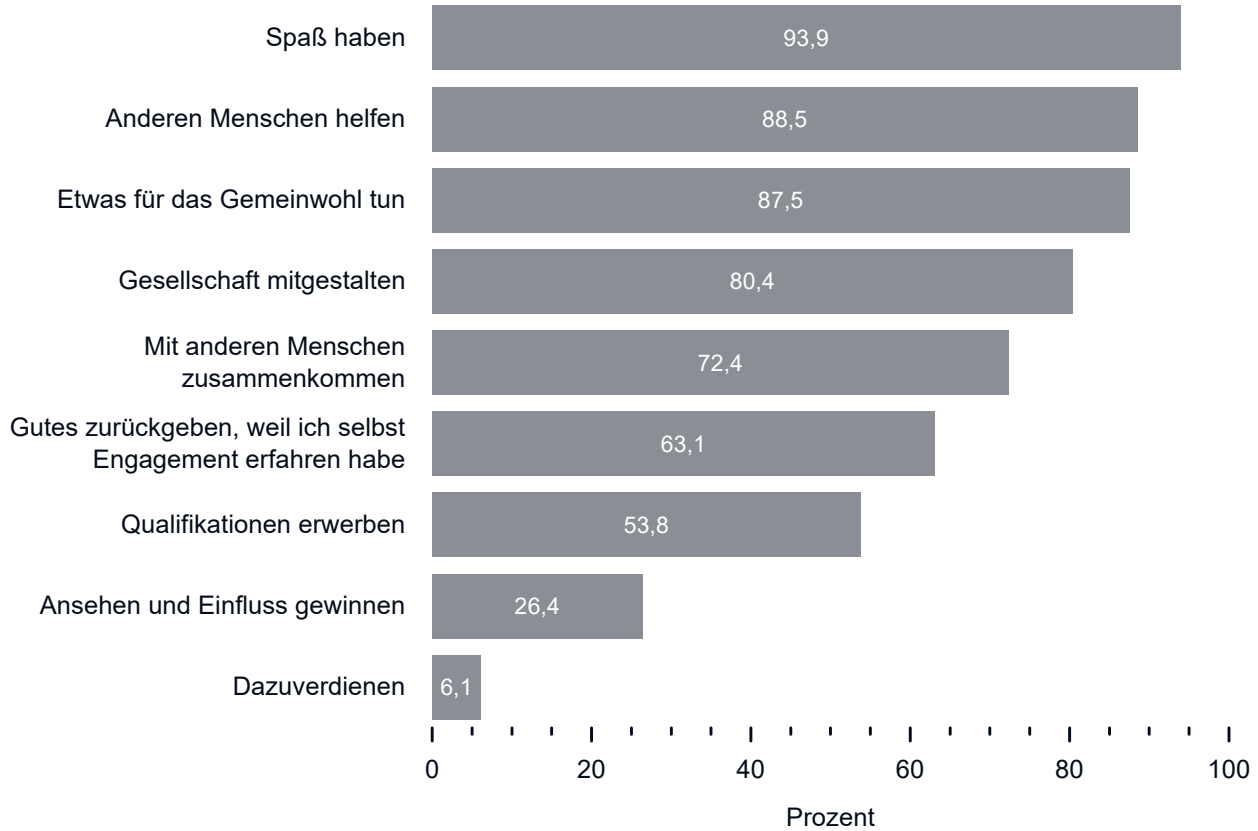

Quelle: FWS 2019, gewichtet, eigene Berechnungen (DZA). Basis: alle Engagierten ( $n=11.813-12.009)$. Mehrfachnennungen möglich. 
ist für mehr als zwei Drittel aller Engagierten (72,4 Prozent) wichtig. Auch die Erfahrung selbst vom Engagement anderer profitiert zu haben, ist für viele Engagierte relevant: 63,1 Prozent von ihnen sagen aus, sie würden ihr Engagement aus diesem Grund betreiben. Persönlicher Gewinn jenseits von Spaß und sozialer Einbettung scheint für Engagierte ebenfalls ein relevantes Motiv zu sein, welches allerdings den eben genannten nachgeordnet wird. Etwas mehr als die Hälfte aller freiwillig Engagierten (53,8 Prozent) möchte im Engagement Qualifikationen erwerben, die im privaten und/oder beruflichen Leben weiterhelfen können. Lediglich 26,4 Prozent aller freiwillig Engagierten sehen das Engagement als etwas an, mit dem sie Ansehen und Einfluss gewinnen können. Das Engagement scheint nur für eine geringe Anzahl der freiwillig Engagierten - nämlich 6,1 Prozent - als Gelegenheit für einen Zuverdienst attraktiv zu sein.

Freiwillig engagierte Frauen und Männer unterscheiden sich hinsichtlich ihrer Motive für ihr Engagement nur geringfügig (Abbildung 6-3). Die Rangfolge der Zustimmung zu den neun abgefragten Motiven ist bei beiden Geschlechtern identisch.

Abbildung 6-3 Angaben der freiwillig Engagierten zu den Motiven für ihr Engagement 2019, nach Geschlecht (Basis: alle Engagierten; Mehrfachnennungen möglich)

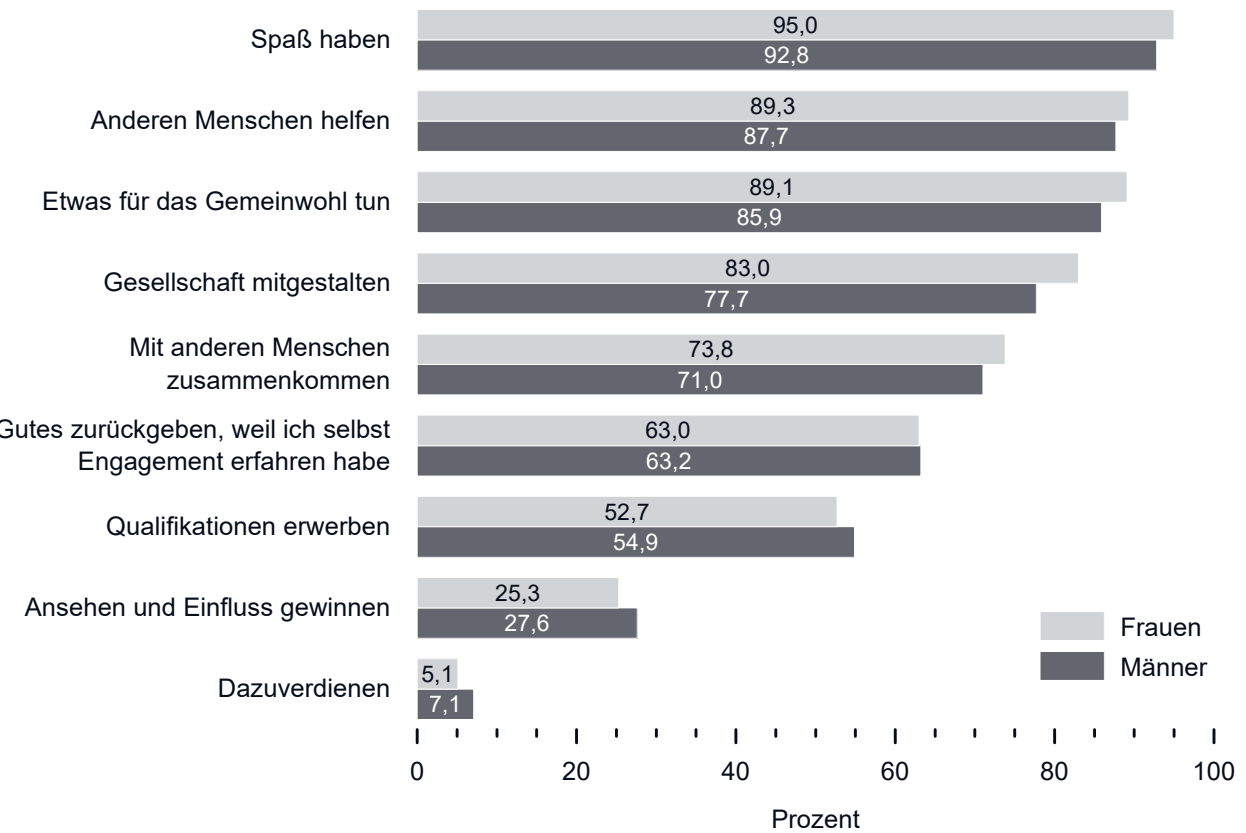

Quelle: FWS 2019, gewichtet, eigene Berechnungen (DZA). Basis: alle Engagierten ( $n=11.813-12.009)$. Mehrfachnennungen möglich.

Die geschlechtsspezifischen Unterschiede sind für die Motive "Spaß haben“, ,Etwas für das Gemeinwohl tun”, „Gesellschaft mitgestalten“, ,Mit anderen Menschen zusammenkommen" und "Dazuverdienen" statistisch signifikant. Für die restlichen Angaben sind die geschlechtsspezifischen Unterschiede statistisch nicht signifikant. 
Abbildung 6-4 Angaben der freiwillig Engagierten zu den Motiven für ihr Engagement 2019, nach Alter (Basis: alle Engagierten; Mehrfachnennungen möglich)

\section{Spaß haben}

Anderen Menschen helfen

Etwas für das Gemeinwohl tun

Gesellschaft mitgestalten

\section{Mit anderen Menschen} zusammenkommen

Gutes zurückgeben, weil ich selbst Engagement erfahren habe

Qualifikationen erwerben

Ansehen und Einfluss gewinnen

Dazuverdienen
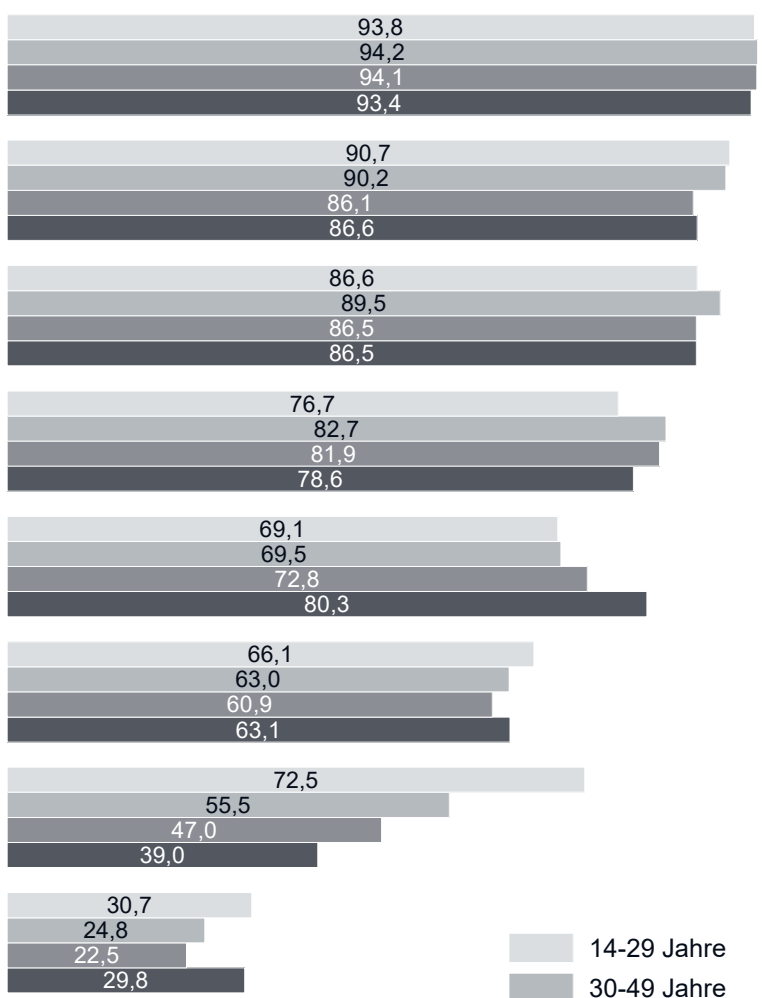

72,5
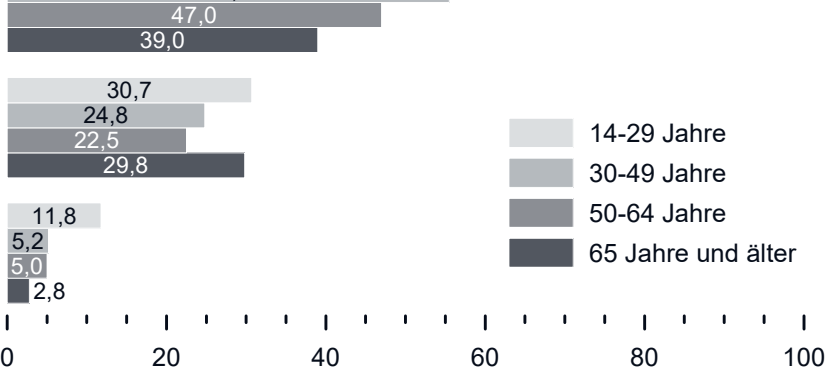

Prozent

Quelle: FWS 2019, gewichtet, eigene Berechnungen (DZA). Basis: alle Engagierten ( $n=11.813-12.009)$. Mehrfachnennungen möglich.

Spaß haben: Kein Unterschied ist statistisch signifikant.

Anderen Menschen helfen: Die Unterschiede zwischen den Gruppen 14-29 Jahre und 50-64 Jahre, 14-29 Jahre und 65 Jahre und älter, 3049 Jahre und 50-64 Jahre sowie 30-49 Jahre und 65 Jahre und älter sind statistisch signifikant.

Etwas für das Gemeinwohl tun: Die Unterschiede für die Gruppe der 30- bis 49-Jährigen zu allen anderen Altersgruppen sind statistisch signifikant.

Gesellschaft mitgestalten: Die Unterschiede zwischen den Gruppen 14-29 Jahre und 30-39 Jahre, 14-29 Jahre und 50-64 Jahre, 30-49 Jahre und 65 Jahre und älter sowie 50-64 Jahre und 65 Jahre und älter sind statistisch signifikant.

Mit anderen Menschen zusammenkommen: Die Unterschiede zwischen allen Altersgruppen, mit Ausnahme desjenigen zwischen den Gruppen 14-29 Jahre und 30-49 Jahre, sind statistisch signifikant.

Gutes zurückgeben, weil ich selbst Engagement erfahren habe: Der Unterschied zwischen den Gruppen 14-29 Jahre und 50-64 Jahre ist statistisch signifikant.

Qualifikationen erwerben: Alle Unterschiede sind statistisch signifikant.

Ansehen und Einfluss gewinnen: Alle Unterschiede mit Ausnahme derjenigen zwischen den Gruppen 14-29 und 65 Jahre und älter sowie 30-49 Jahre und 50-64 Jahre sind statistisch signifikant.

Dazuverdienen: Alle Unterschiede, mit Ausnahme desjenigen zwischen den Gruppen 30-49 Jahre und 50-64 Jahre, sind statistisch signifikant. 
Frauen geben anteilig häufiger als Männer an, ihr Engagement auszuüben, weil sie Spaß daran haben (95,o Prozent zu 92,8 Prozent), die Gesellschaft mitgestalten wollen (83,o Prozent zu 77,7 Prozent), etwas für das Gemeinwohl tun möchten (89,1 Prozent zu 85,9 Prozent) oder mit anderen Menschen zusammenkommen möchten (73,8 Prozent zu 71,o Prozent). Männer üben ihr freiwilliges Engagement im Gegensatz dazu anteilig häufiger als Frauen aus, um etwas dazuzuverdienen (7,1 Prozent zu 5,1 Prozent). In der Zustimmung zu allen anderen Motiven unterscheiden sich Frauen und Männer nicht statistisch signifikant.

Die Unterschiede zwischen Altersgruppen (Abbildung 6-4) hinsichtlich ihrer Motive sind ausgeprägter als die Unterschiede zwischen Frauen und Männern. Für alle Altersgruppen ist der Spaß das am häufigsten und in einem ähnlichen Ausmaß genannte Motiv für ihr Engagement. Für die Engagierten, die 65 Jahre und älter sind, stellt die Gelegenheit mit anderen Menschen zusammenzukommen (8o,3 Prozent), ein deutlich wichtigeres Motiv dar als für alle anderen Gruppen. Weiterhin ist es für Engagierte dieser Altersgruppe vergleichsweise häufig ein Motiv, mit dem Engagement Ansehen und Einfluss zu gewinnen (29,8 Prozent). Nur für die jüngste Altersgruppe der 14- bis 29-Jährigen hat dieses Motiv einen ähnlich hohen Stellenwert. Für die Gruppe der 14- bis 29-Jährigen ist es zudem wichtig, durch ihr freiwilliges Engagement Qualifikationen erwerben zu können ( 72,5 Prozent). Dieses Motiv zeigt die größten altersspezifischen Unterschiede, wobei die Relevanz für die Befragten mit höherem Alter anteilig deutlich geringer ist. Zudem geben 11,8 Prozent der Engagierten in der jüngsten Altersgruppe an, die Möglichkeit des Dazuverdienens motiviere sie. Die jüngste Altersgruppe weist zusammen mit der zweitjüngsten, den 30- bis 49-Jährigen, auch die stärkste Zustimmung zum Motiv anderen zu helfen auf. Den 30- bis 49-Jährigen ist zudem im Vergleich zu den anderen Altersgruppen am häufigsten daran gelegen etwas für das Gemeinwohl zu tun (89,5 Prozent). Das Motiv die Gesellschaft mitzugestalten findet sich bei beiden mittleren Altersgruppen, den 30bis 49-Jährigen und den 50- bis 64-Jährigen, anteilig am häufigsten. Das Motiv ein Engagement auszuüben, weil die Person selbst Nutznießende des Engagements von anderen gewesen ist, ist in allen Altersgruppen ähnlich ausgeprägt, wobei die 14- bis 29-Jährigen dieses Motiv etwas häufiger angeben als die 50- bis 64-Jährigen.

\subsection{Beendigungsgründe für Engagement}

Als Beendigungsgrund für ein freiwilliges Engagement werden berufliche Gründe mit 43,3 Prozent anteilig am häufigsten angeführt (Abbildung 6-5). Insgesamt 42,9 Prozent der ehemals freiwillig Engagierten geben an, ihr Engagement beendet zu haben, weil die Tätigkeit zeitlich befristet war. Für 40,4 Prozent der ehemals Engagierten führt der zeitliche Aufwand zur Beendigung des freiwilligen Engagements. 31,5 Prozent beenden ihr Engagement, um keine Verpflichtung mehr zu haben. Familiäre Gründe führen bei 26,6 Prozent der vormals freiwillig Engagierten zu einer 
Abbildung 6-5 Beendigungsgründe für früheres Engagement 2019 (Basis: früher engagierte Personen; Mehrfachnennungen möglich)

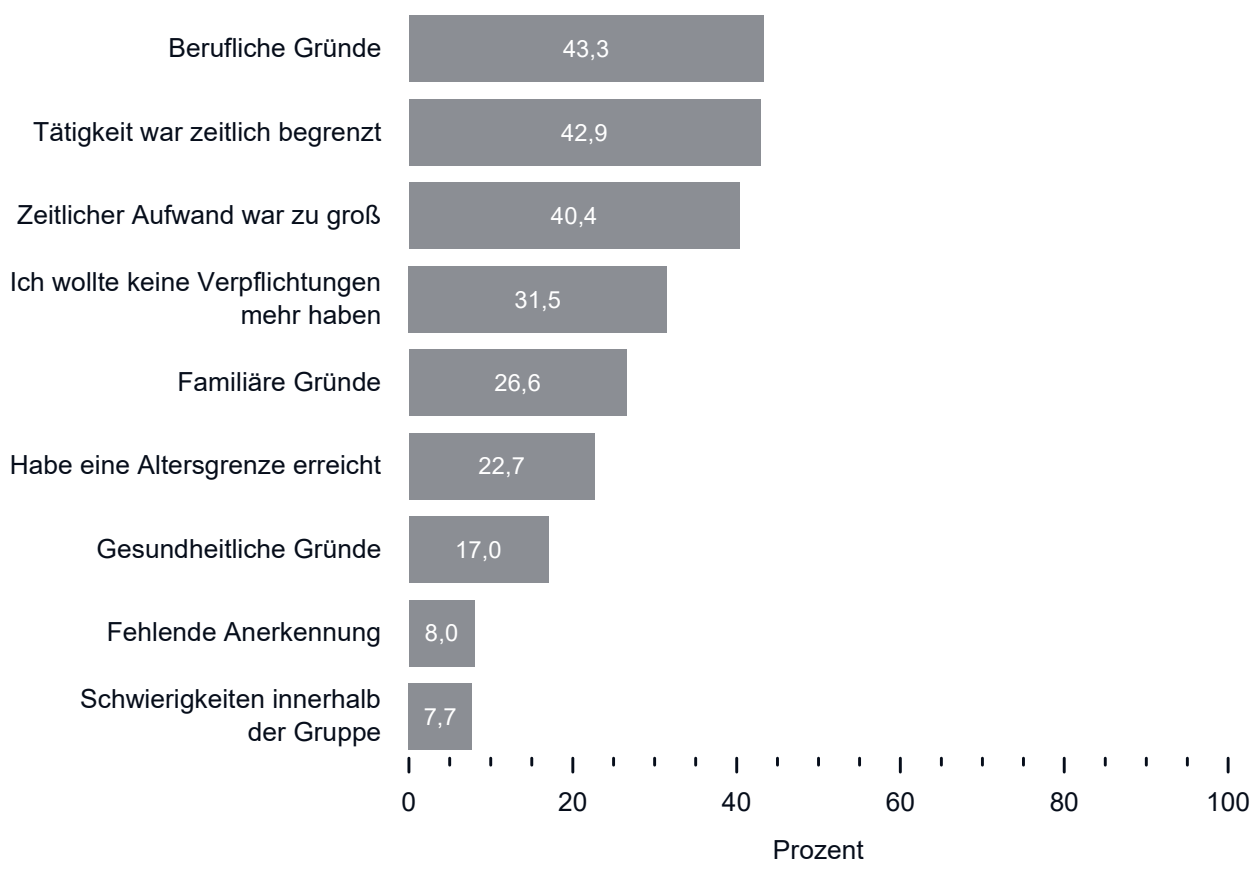

Quelle: FWS 2019, gewichtet, eigene Berechnungen (DZA). Basis: früher engagierte Personen, die zurzeit nicht freiwillig engagiert sind $(n=6.705-6.737)$. Mehrfachnennungen möglich.

Beendigung ihrer Tätigkeit. 22,7 Prozent geben an, aufgrund einer Altersgrenze ihr Engagement zu beenden und 17,0 Prozent beenden ihr Engagement aus gesundheitlichen Gründen. Negative Erfahrungen während des Engagements, wie etwa fehlende Anerkennung mit 8,o Prozent und Schwierigkeiten in der Gruppe mit 7,7 Prozent, scheinen im Vergleich, eher nachrangige Beendigungsgründe zu sein.

Bei Frauen spielen familiäre Gründe eine deutlich größere Rolle bei der Beendigung des Engagements als bei Männern (33,5 Prozent zu 19,4 Prozent; Abbildung 6-6). Zudem geben Frauen anteilig häufiger als Männer an, ihr freiwilliges Engagement aus gesundheitlichen Gründen oder aufgrund einer zeitlichen Befristung zu beenden. Männer hingegen beenden ihr Engagement eher aus beruflichen Gründen als Frauen (46,7 Prozent zu 40,o Prozent). Zudem geben Männer anteilig häufiger als Frauen an, dass Schwierigkeiten innerhalb der Gruppe zu einer Beendigung ihrer freiwilligen Tätigkeit führen (9,8 Prozent zu 5,7 Prozent). Demgegenüber stellen ein hoher zeitlicher Aufwand, der Wunsch, keine Verpflichtung mehr zu haben, das Erreichen einer bestimmten Altersgrenze sowie fehlende Anerkennung ihres Engagements für Männer und Frauen in gleichem Ausmaß Beendigungsgründe dar. 
Abbildung 6-6 Beendigungsgründe für früheres Engagement 2019, nach Geschlecht (Basis: früher engagierte Personen; Mehrfachnennungen möglich)

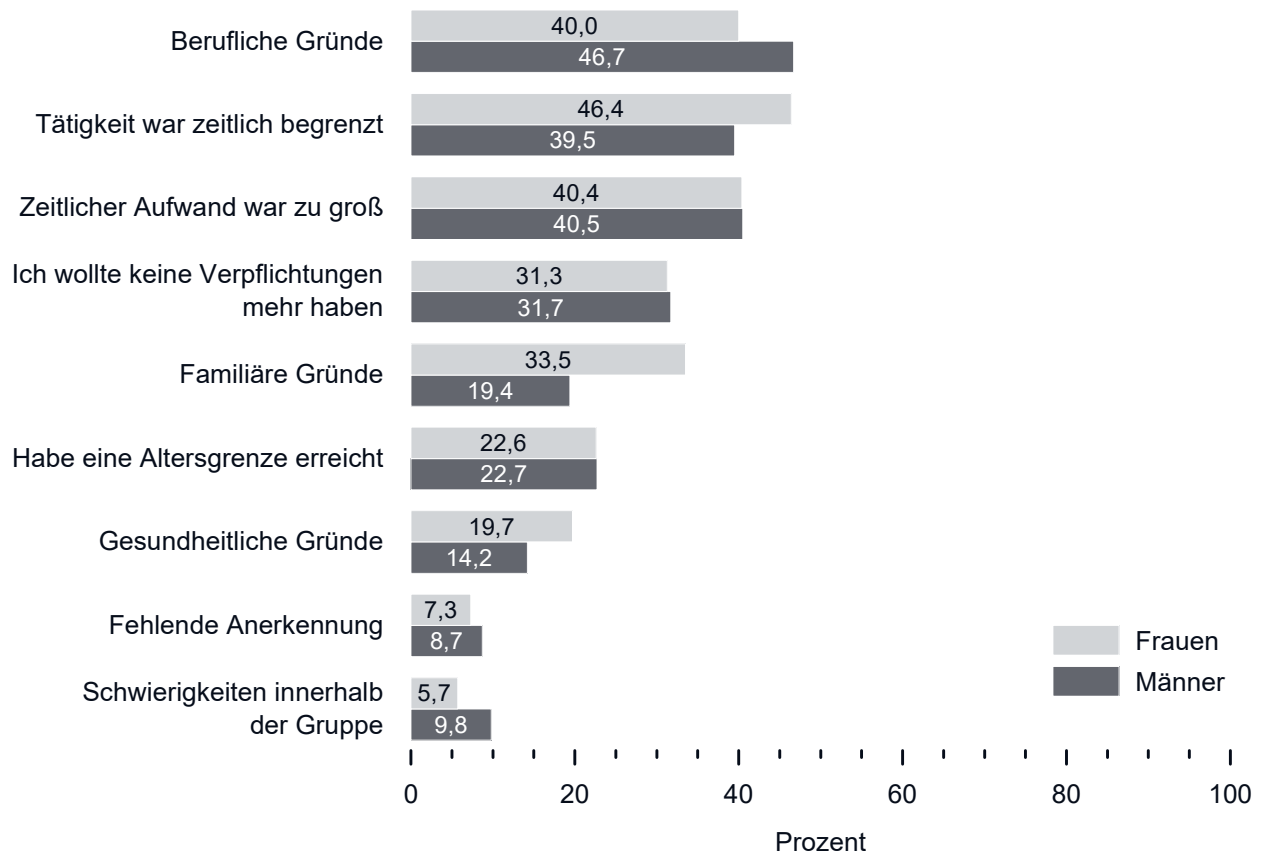

Quelle: FWS 2019, gewichtet, eigene Berechnungen (DZA). Basis: früher engagierte Personen, die zurzeit nicht freiwillig engagiert sind ( $n=6.705-6.737)$. Mehrfachnennungen möglich.

Die geschlechtsspezifischen Unterschiede sind für die Beendigungsgründe „BBerufliche Gründe“, ,'Tätigkeit war zeitlich begrenzt", ${ }^{\prime}$ Familiäre Gründe", "Gesundheitliche Gründe" und "Schwierigkeiten innerhalb der Gruppe" statistisch signifikant. Für die restlichen Beendigungsgründe sind die geschlechtsspezifischen Unterschiede statistisch nicht signifikant.

Unterschiede zwischen Altersgruppen liegen nicht für alle Beendigungsgründe vor (Abbildung 6-7). So finden sich fehlende Anerkennung und Schwierigkeiten innerhalb der Gruppe in allen Altersgruppen in einem vergleichbaren Ausmaß. Die Gruppe der 30- bis 49-Jährigen beendet ihr freiwilliges Engagement anteilig am häufigsten aus beruflichen oder allgemein aus zeitlichen Gründen. Familiäre Gründe sind insbesondere in den beiden Altersgruppen der Personen, die 50 Jahre oder älter sind, relevante Beendigungsgründe. Dieser Grund wird von Personen im Alter zwischen 14 und 29 Jahren anteilig deutlich seltener genannt. Auch der Wunsch keine Verpflichtungen mehr zu haben, führt in den Altersgruppen der Personen ab 50 Jahren anteilig häufiger zur Beendigung ihres Engagements. Erwartungsgemäß spielen gesundheitliche Gründe in diesen beiden Gruppen ebenfalls eine stärkere Rolle als bei den jüngeren Altersgruppen und der Einfluss der Gesundheit ist in der ältesten Gruppe noch deutlicher ausgeprägt als bei den 50- bis 64-Jährigen. Zudem geben ehemals Engagierte ab 65 Jahren mit Abstand am anteilig häufigsten an, sie hätten ihr Engagement beendet, weil sie eine Altersgrenze erreicht haben. 


\section{Abbildung 6-7 Beendigungsgründe für früheres Engagement 2019, nach Alter (Basis: früher engagierte Personen; Mehrfachnennungen möglich)}

Berufliche Gründe

Tätigkeit war zeitlich begrenzt

Zeitlicher Aufwand war zu groß

Ich wollte keine Verpflichtungen mehr haben

Familiäre Gründe

Habe eine Altersgrenze erreicht

Gesundheitliche Gründe Fehlende Anerkennung Schwierigkeiten innerhalb der Gruppe
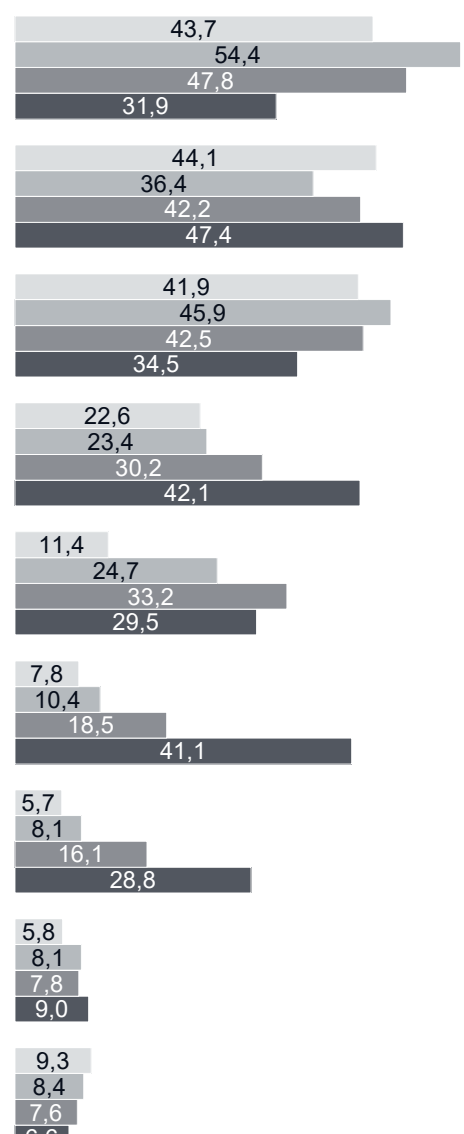

9,3
8,4
7,6 6,6

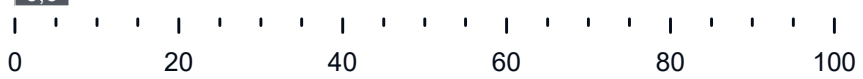

Prozent

Quelle: FWS 2019, gewichtet, eigene Berechnungen (DZA). Basis: früher engagierte Personen, die zurzeit nicht freiwillig engagiert sind $(\mathrm{n}=6.705-6.737)$. Mehrfachnennungen möglich.

Berufliche Gründe: Alle Unterschiede, mit Ausnahme desjenigen zwischen den Gruppen 14-29 Jahre und 50-64 Jahre, sind statistisch signifikant.

Tätigkeit war zeitlich begrenzt: Alle Unterschiede, mit Ausnahme derjenigen zwischen den Gruppen 14-29 Jahre und 50-64 Jahre sowie 1429 Jahre und 65 Jahre und älter, sind statistisch signifikant.

Zeitlicher Aufwand war zu groß: Die Unterschiede für die Gruppe 65 Jahre und älter zu allen anderen Altersgruppen sind statistisch signifikant.

Ich wollte keine Verpflichtungen mehr haben: Alle Unterschiede, mit Ausnahme desjenigen zwischen den Altersgruppen 14-29 Jahre und 30-49 Jahre, sind statistisch signifikant.

Familiäre Gründe: Alle Unterschiede, mit Ausnahme desjenigen zwischen den Gruppen 50-64 Jahre und 65 Jahre und älter, sind statistisch signifikant.

Habe eine Altersgrenze erreicht: Alle Unterschiede, mit Ausnahme desjenigen zwischen den Altersgruppen 14-29 Jahre und 30-49 Jahre, sind statistisch signifikant.

Gesundheitliche Gründe: Alle Unterschiede, mit Ausnahme desjenigen zwischen den Altersgruppen 14-29 Jahre und 30-49 Jahre, sind statistisch signifikant.

Fehlende Anerkennung: Kein Unterschied ist statistisch signifikant.

Schwierigkeiten innerhalb der Gruppe: Kein Unterschied ist statistisch signifikant. 


\subsection{Hinderungsgründe für Engagement}

Die überwiegende Mehrheit von 71,3 Prozent der noch nie Engagierten nennt zeitliche Einschränkungen als eine Ursache, die das Aufnehmen eines Engagements verhindert (Abbildung 6-8). Berufliche Gründe werden von 41,4 Prozent der nie engagierten Personen anteilig am zweithäufigsten genannt. 37,9 Prozent möchten keine Verpflichtungen eingehen. Familiäre Gründe werden von 29,3 Prozent und gesundheitliche Gründe von 25,5 Prozent als Gründe angeführt, bisher kein freiwilliges Engagement aufgenommen zu haben. Ein substanzieller Anteil der noch nie Engagierten, 23,1 Prozent, gibt an, nicht zu wissen, wohin man sich wenden könne, um eine freiwillige Tätigkeit aufzunehmen. Etwas mehr als ein Fünftel (21,6 Prozent) fühlt sich für ein Engagement nicht geeignet. Lediglich ein geringer Anteil nie engagierter Personen (2,6 Prozent) erklärt, sich um ein Engagement bemüht zu haben, aber abgelehnt worden zu sein.

Bei Personen, die nie ein Engagement ausgeübt haben, sind die Geschlechterunterschiede bei den meisten Hinderungsgründen gering (Abbildung 6-9). Auffällig

Abbildung 6-8 Hinderungsgründe für freiwilliges Engagement 2019 (Basis: nie engagierte Personen; Mehrfachnennungen möglich)

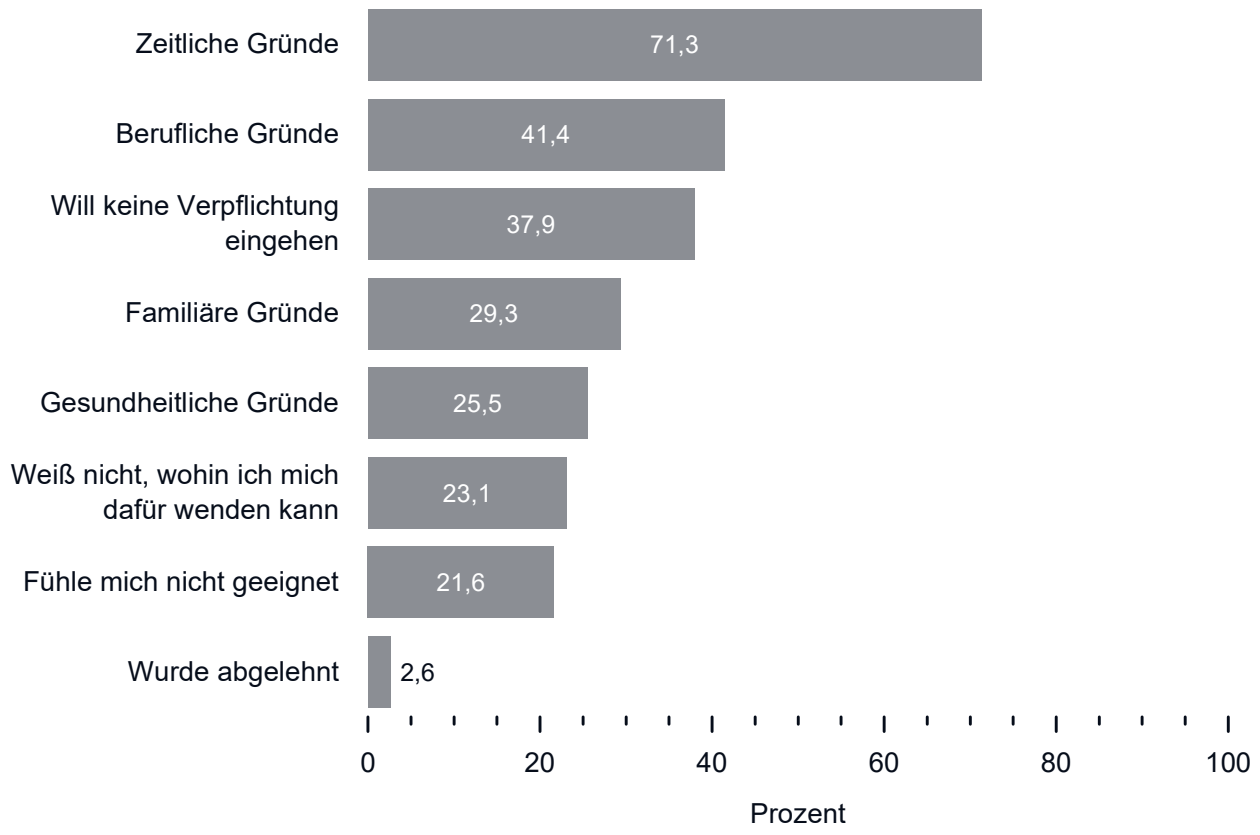

Quelle: FWS 2019, gewichtet, eigene Berechnungen (DZA). Basis: bislang noch nie engagierte Personen $(n=8.526-8.639)$. Mehrfachnennungen möglich. 
Abbildung 6-9 Hinderungsgründe für freiwilliges Engagement 2019, nach Geschlecht (Basis: nie engagierte Personen; Mehrfachnennungen möglich)

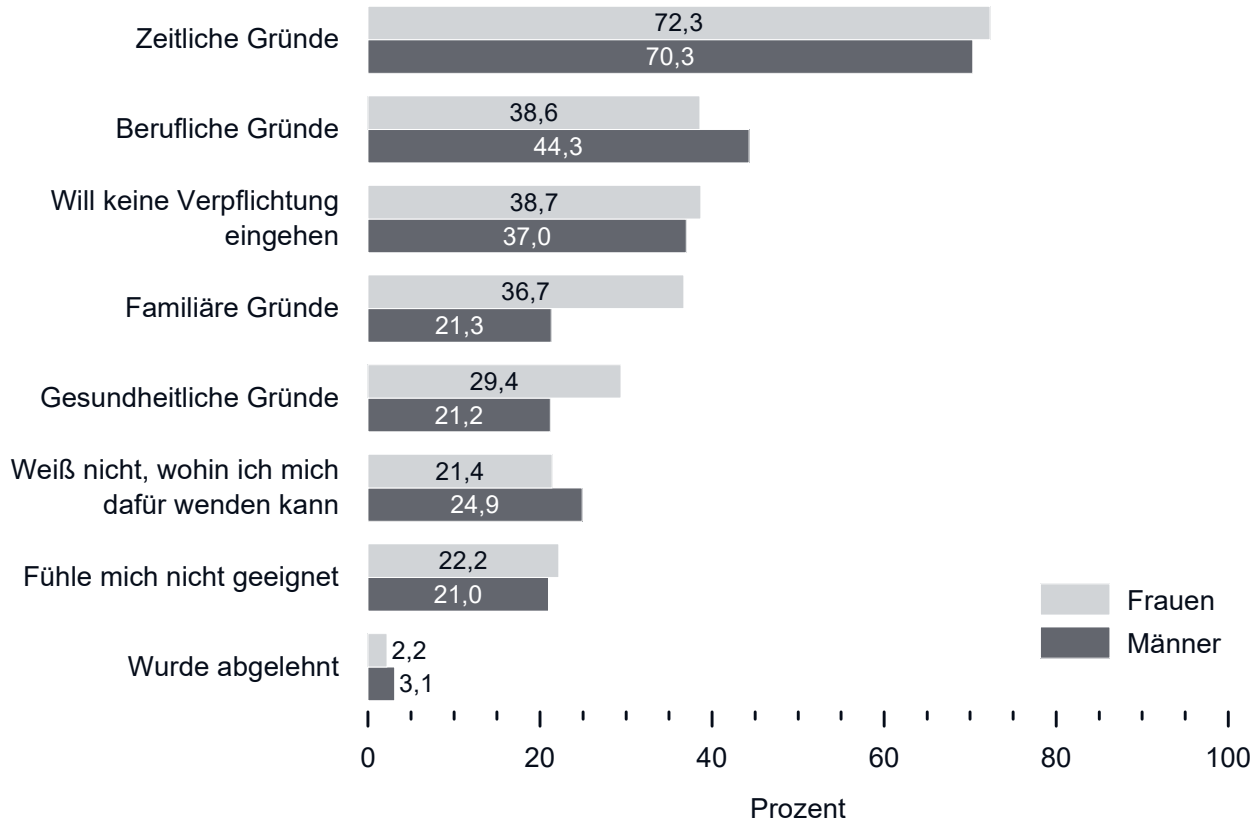

Quelle: FWS 2019, gewichtet, eigene Berechnungen (DZA). Basis: bislang noch nie engagierte Personen $(\mathrm{n}=8.526-8.639)$. Mehrfachnennungen möglich.

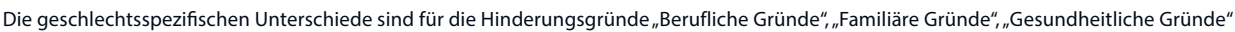
und "Weiß nicht, wohin ich mich dafür wenden kann" statistisch signifikant. Für die restlichen Hinderungsgründe sind die Unterschiede statistisch nicht signifikant.

ist, dass Frauen anteilig deutlich häufiger als Männer familiäre Gründe nennen, die sie von der Aufnahme eines Engagements abhalten (36,7 Prozent zu 21,3 Prozent). Ebenso nennen anteilig mehr Frauen als Männer gesundheitliche Gründe (29,4 Prozent zu 21,2 Prozent). Männer dagegen geben anteilig etwas häufiger berufliche Gründe als Hinderungsgrund an (44,3 Prozent zu 38,6 Prozent). Zudem geben Männer anteilig etwas häufiger als Frauen an, nicht zu wissen, wohin sie sich für ein Engagement wenden könnten (24,9 Prozent zu 21,4 Prozent). Keine geschlechtsspezifischen Unterschiede gibt es bei zeitlichen Gründen, dem Wunsch, keine Verpflichtung eingehen zu wollen, dem Gefühl ungeeignet zu sein sowie einer Ablehnung.

Wie in Abbildung 6-10 dargestellt, unterscheiden sich die Hinderungsgründe für die Aufnahme eines Engagements zwischen den Altersgruppen. Auffällig ist, dass Personen, die noch nie engagiert waren und 50 Jahre und älter sind, anteilig besonders häufig angeben, keine Verpflichtungen eingehen zu wollen. 41,5 Prozent der 50bis 64-Jährigen sowie 48,7 Prozent der Menschen im Alter von 65 Jahren und älter nehmen aus diesem Grund kein freiwilliges Engagement auf. Zudem stellen gesund- 


\section{Abbildung 6-10 Hinderungsgründe für freiwilliges Engagement 2019, nach Alter (Basis: nie engagierte Personen; Mehrfachnennungen möglich)}

Zeitliche Gründe

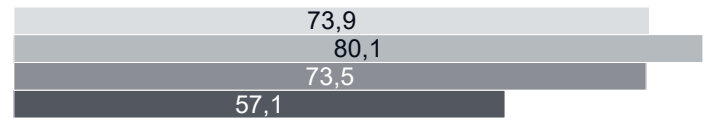

Berufliche Gründe

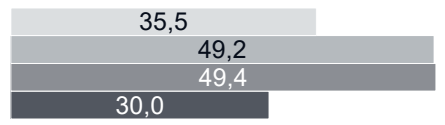

Will keine Verpflichtung eingehen

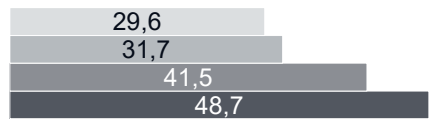

Familiäre Gründe

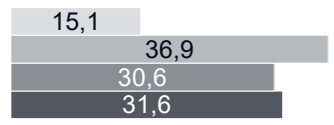

Gesundheitliche Gründe

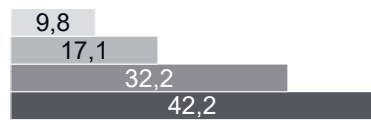

Weiß nicht, wohin ich mich dafür wenden kann

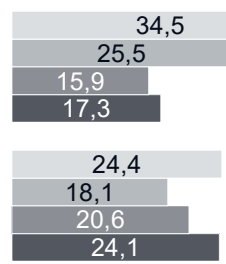

4-29 Jahre

Fühle mich nicht geeignet 30-49 Jahre

\section{4,3}

Wurde abgelehnt

$\begin{array}{r}2,2 \\ 2,1 \\ 2,2 \\ 1 \\ 1 \\ \hline\end{array}$
20 40 60 80

Prozent

Quelle: FWS 2019, gewichtet, eigene Berechnungen (DZA). Basis: bislang noch nie engagierte Personen ( $\mathrm{n}=8.526-8.639)$. Mehrfachnennungen möglich.

Zeitliche Gründe: Alle Unterschiede, mit Ausnahme desjenigen zwischen den Gruppen 14-29 Jahre und 50-64 Jahre, sind statistisch signifikant.

Berufliche Gründe: Alle Unterschiede, mit Ausnahme desjenigen zwischen den Gruppen 30-49 Jahre und 50-64 Jahre, sind statistisch signifikant.

Will keine Verpflichtung eingehen: Alle Unterschiede, mit Ausnahme desjenigen zwischen den Gruppen 14-29 Jahre und 30-49 Jahre, sind statistisch signifikant.

Familiäre Gründe: Alle Unterschiede, mit Ausnahme desjenigen zwischen den Gruppen 50-64 Jahre und 65 Jahre und älter, sind statistisch signifikant.

Gesundheitliche Gründe: Alle Unterschiede sind statistisch signifikant.

Weiß nicht, wohin ich mich dafür wenden kann: Alle Unterschiede, mit Ausnahme desjenigen zwischen den Gruppen 50-64 Jahre und 65 Jahre und älter, sind statistisch signifikant.

Fühle mich nicht geeignet: Die Unterschiede zwischen den Gruppen 14-29 Jahre und 30-39 Jahre sowie 30-49 Jahre und 65 Jahre und älter sind statistisch signifikant.

Wurde abgelehnt: Die Unterschiede für die Gruppe 14-29 Jahre zu allen anderen Altersgruppen sind statistisch signifikant. 
heitliche Gründe in den höheren Altersgruppen einen wichtigen Hinderungsgrund dar. 32,2 Prozent in der Gruppe der 50- bis 64-Jährigen und 42,2 Prozent bei den Menschen ab 65 Jahren sehen gesundheitliche Gründe als Hinderungsgrund an. In der jüngsten Altersgruppe der 14- bis 29-Jährigen werden vergleichsweise wenig familiäre, berufliche und gesundheitliche Gründe genannt. Aber die Personen dieser Altersgruppe geben anteilig verhältnismäßig häufig an, nicht zu wissen, wohin sie sich wenden können (34,5 Prozent) und fühlen sich anteilig ebenso häufig wie Personen der höchsten Altersgruppe nicht für ein Engagement geeignet (24,4 Prozent). Für die Gruppe der 30- bis 49-Jährigen sind insbesondere zeitliche, berufliche und familiäre Gründe Ursachen dafür, dass sie bislang kein Engagement aufgenommen haben.

\subsection{Engagementbereitschaft}

Die Engagementbereitschaft gibt Aufschluss über das Potenzial, weitere Menschen für ein freiwilliges Engagement zu gewinnen. Hierbei werden sowohl Personen, die noch nie freiwillig engagiert waren, als auch Personen, die in der Vergangenheit engagiert waren und ihr freiwilliges Engagement beendet haben, nach ihrer Bereitschaft gefragt, in Zukunft ein freiwilliges Engagement aufzunehmen (Gruppen b und c der Abbildung 6-1). Insgesamt lässt sich feststellen, dass die Mehrheit von 58,7 Prozent aller Personen, die 2019 nicht engagiert sind, sich vorstellen kann, in Zukunft ein freiwilliges Engagement aufzunehmen. Die Engagementbereitschaft ist insgesamt zwischen 1999 und 2019 stetig angestiegen. Der Anteil derzeit nicht-engagierter Menschen, die sich die Aufnahme einer freiwilligen Tätigkeit vorstellen können, betrug 38,7 Prozent im Jahr 1999, 49,4 Prozent im Jahr 2004, 55,1 Prozent im Jahr 2009 und 56,9 Prozent im Jahr 2014 (ohne Abbildung). Dies entspricht einem Anstieg von 20 Prozentpunkten zwischen den Jahren 1999 und 2019. Der beobachtete Anstieg ist zwischen allen Erhebungszeitpunkten statistisch signifikant. Vergleiche zwischen den Jahren 1999, 2004, 2009 und 2014 und 2019 müssen allerdings sehr vorsichtig interpretiert werden, da die Abfrage im Jahr 2014 konkretisiert wurde (siehe Abschnitt 6.2). Inhaltlich gleiche Fragen können aufgrund unterschiedlicher Formulierungen von den Befragten unterschiedlich verstanden werden.

2019 können sich aktuell nicht-engagierte Frauen die Aufnahme eines Engage-

ments anteilig seltener vorstellen als nicht-engagierte Männer (Abbildung 6-11). Auffällig ist das starke Gefälle der Engagementbereitschaft 2019 über die Altersgruppen. Während in der jüngsten Altersgruppe von 14 bis 29 Jahren eine Mehrheit von 82,4 Prozent, bei den Menschen im Alter von 30 bis 49 Jahren immer noch 75, o Prozent und bei den Befragten im Alter zwischen 50 und 64 Jahren 59,3 Prozent bereit sind, eine freiwillige Tätigkeit aufzunehmen, sind es in der höchsten Altersgruppe der 65-Jährigen und Älteren noch 27,2 Prozent, die prinzipiell ein Engagement aufnehmen würden. 
Abbildung 6-11 Anteile aktuell nicht-engagierter Personen, die zu einem Engagement bereit sind, 2019, gesamt, nach Geschlecht und nach Alter (Basis: aktuell nicht-engagierte Personen)

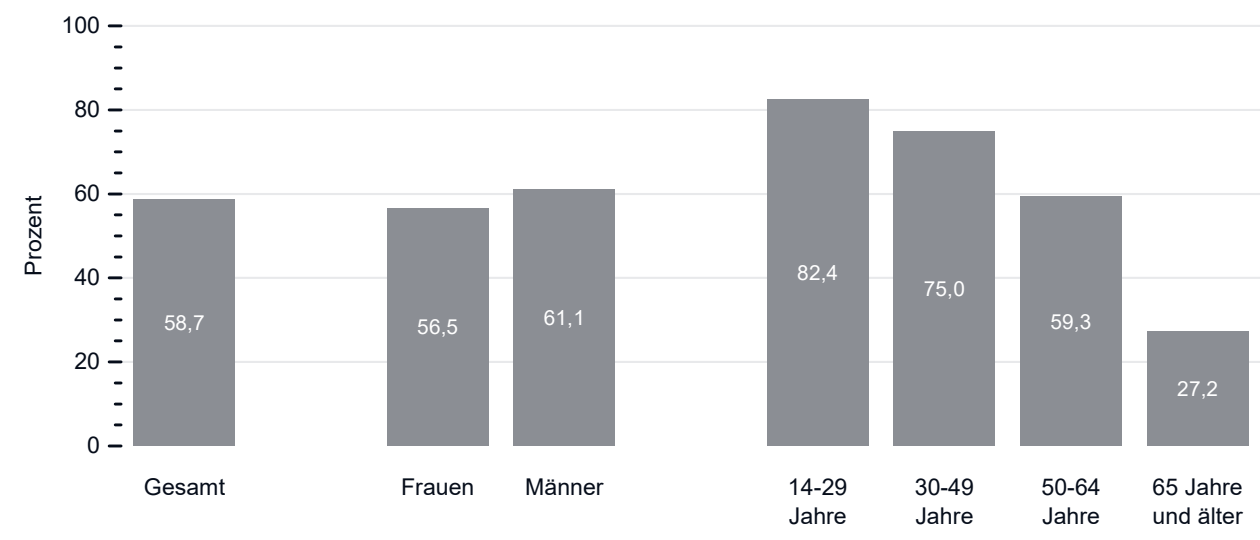

Quelle: FWS 2019, gewichtet, eigene Berechnungen (DZA). Basis: aktuell nicht-engagierte Personen ( $n=15.479)$.

Die geschlechts- sowie alle altersspezifischen Unterschiede sind statistisch signifikant.

\subsection{Fazit}

In diesem Kapitel wurde über die individuellen Motive für das Engagement bei freiwillig Engagierten, die Gründe für die Beendigung eines Engagements bei ehemals Engagierten, die Hinderungsgründe für die Ausübung eines Engagements bei Personen, die niemals engagiert waren sowie die Engagementbereitschaft aktuell nichtengagierter Personen, berichtet. Aus der Darstellung dieser unterschiedlichen Aspekte und deren Zusammenhänge mit den untersuchten Indikatoren lassen sich Erkenntnisse gewinnen, die zur Schaffung besserer Voraussetzungen für freiwilliges Engagement beitragen könnten. Verbesserte Rahmenbedingungen für freiwilliges Engagement könnten zum Abbau der Unterschiede in der gesellschaftlichen Teilhabe zwischen den Geschlechtern und zwischen den verschiedenen Altersgruppen führen und Engagement vieler Menschen über einen möglichst langen Zeitraum und mit hoher Zufriedenheit ermöglichen.

Die Motive für Engagement können altruistischer, sozialer oder eigennütziger Natur sein, wobei die Engagierten den sozialen und altruistischen Motiven eine höhere Bedeutung beimessen. Das anteilig am häufigsten genannte Motiv für ein Engagement ist der Spaß, den die Engagierten bei ihren freiwilligen Tätigkeiten empfinden. Die im Freiwilligensurvey 2019 neu aufgenommenen Motive, anderen zu helfen und etwas für das Gemeinwohl zu tun, sind die am zweit- und dritthäufigsten genannten Motive. Für fast neun von zehn Engagierten sind diese altruistischen Motive wichtig.

Als Beendigungs- und Hinderungsgründe für das Engagement werden häufig Gründe genannt, die nicht direkt mit dem Engagement zusammenhängen, sondern aus anderen Lebenslagen resultieren. Berufliche und zeitliche Gründe werden als 
Hauptursachen genannt, weshalb ein Engagement beendet wird. Zugleich stellen zeitliche Gründe den mit Abstand am häufigsten genannten Grund dar, weshalb ehemals Engagierte ihr Engagement beenden und Menschen, die noch nie engagiert waren, kein Engagement aufnehmen. Diese Gründe könnten durch verbesserte Rahmenbedingungen abgeschwächt werden: Zeitlich flexibel organisierte Engagementtätigkeiten könnten Zugänge zum Engagement und die Aufrechterhaltung der Tätigkeiten im Engagement erleichtern. Der Einsatz von digitalen Technologien könnte weitere zeitliche Hindernisse in Bezug auf die Aufnahme oder Aufrechterhaltung eines Engagements abbauen, indem beispielsweise Fahrzeiten reduziert oder auch schnellere Kommunikationswege genutzt werden.

Insgesamt scheint die Bereitschaft für ein freiwilliges Engagement groß zu sein. Immerhin geben 58,7 Prozent aller im Jahre 2019 nicht-engagierten Personen an, sich prinzipiell für eine freiwillige Tätigkeit zu interessieren. Um dieses Potenzial zu nutzen, müssen bei der Rekrutierung neuer Engagierter sowohl die unterschiedlichen Motive als auch die Unterschiede in den Beendigungs- und Hinderungsgründen, die zwischen den Geschlechtern und Altersgruppen bestehen, von den Organisationen des Engagements berücksichtigt werden. Bei den verschiedenen Kampagnen muss aber auch sichergestellt werden, dass die Online-Plattformen für Engagementinteressierte einfach zu finden sowie leicht verständlich und zugänglich sind, um eine möglichst große Gruppe zu erreichen. Da Internetzugang und -kompetenzen in der Gesellschaft sehr unterschiedlich verteilt sind, ist es wichtig, Offline-Kampagnen nicht zu vernachlässigen, um auf diese Weise auch Personengruppen zu erreichen, die über keinen Zugang zum Internet oder zu wenige entsprechende Kompetenzen verfügen (Deutscher Bundestag 2020).

In den Ergebnissen sehen wir, dass sich Frauen und Männer in ihren Motiven für ein Engagement relativ wenig unterscheiden. Auffällige Geschlechterunterschiede finden sich dagegen in den Beendigungs- und den Hinderungsgründen für eine freiwillige Tätigkeit. Insbesondere familiäre Gründe werden von Frauen anteilig häufiger als Beendigungs- und Hinderungsgründe genannt als von Männern. Frauen sind nicht nur stärker als Männer in die Kinderbetreuung eingebunden, sondern übernehmen im mittleren und späten Erwachsenenalter auch häufiger als Männer Aufgaben in der Pflege und Unterstützung von gesundheitlich eingeschränkten Angehörigen und in der Enkelkinderbetreuung (Klaus \& Vogel 2019). Zwar nennen Männer anteilig häufiger als Frauen berufliche Gründe als Ursache für die Beendigung ihres Engagements (46,7 Prozent), aber auch zwei von fünf der ehemals engagierten Frauen, sagen aus, sie hätten ihre freiwillige Tätigkeit aus beruflichen Gründen beendet. Eine zeitliche Flexibilisierung der Engagementaufgaben sowie eine Verbesserung der Vereinbarkeit von Familie, Beruf und freiwilligem Engagement könnten das Engagement für Frauen ebenso wie für Männer in unterschiedlichen Lebensphasen erleichtern.

Unterschiede zwischen Altersgruppen gibt es bei den Beendigungs- und Hinderungsgründen sowie den Motiven und der Engagementbereitschaft. Die Engage- 
mentbereitschaft ist in der jüngsten Altersgruppe besonders hoch. Eine überwiegende Mehrheit (82,4 Prozent) der Personen im Alter von 14 bis 29 Jahren, die zum Befragungszeitpunkt nicht engagiert waren, kann sich prinzipiell vorstellen ein Engagement aufzunehmen. Um dieses hohe Potenzial bei der Rekrutierung freiwillig Engagierter zu nutzen, können unter Rückbezug auf die Motive sowie die Beendigungsund Hinderungsgründe das Engagement für diese Altersgruppe attraktiver gestaltet und bestehende Barrieren, wie beispielsweise zeitliche Gründe oder die Unkenntnis darüber, wohin sie sich dafür wenden können, abgebaut werden. Im Vergleich aller Altersgruppen sind für engagierte Personen im Alter von 14 bis 29 Jahren die Motive, sich weiter zu qualifizieren, Ansehen zu gewinnen und auch etwas dazuzuverdienen vergleichsweise wichtig. Gleichzeitig zweifeln noch nie Engagierte dieser Altersgruppe auch eher an ihrer Eignung und geben anteilig deutlich häufiger als alle anderen Altersgruppen an, sich um ein Engagement bemüht zu haben, jedoch abgelehnt worden zu sein. Eine verstärkte Informationsarbeit, die Zugangsmöglichkeiten zum Engagement klar darlegt und sich direkt an jüngere Menschen richtet, könnte hier Abhilfe schaffen. Eine solche Kampagne sollte zudem insbesondere die Vorteile des Engagements für die persönliche Weiterentwicklung wie etwa die Möglichkeiten zur Weiterqualifizierung herausstellen. Beispielsweise die Kampagne „Deine Zeit ist jetzt“ (Bundesanstalt Technisches Hilfswerk 2020) des Technischen Hilfswerks hebt diesen Aspekt der freiwilligen Tätigkeit besonders hervor und bietet Weiterbildungen in verschiedenen Bereichen, wie etwa Arbeitssicherheit, Didaktik oder Presseund Öffentlichkeitsarbeit, an.

Engagierte Menschen im mittleren Lebensalter zwischen 30 und 49 Jahren nennen anteilig besonders häufig altruistische Motive, wie etwa anderen zu helfen oder etwas für das Gemeinwohl zu tun, als Gründe für ihr freiwilliges Engagement. Allerdings stellen die besonderen Lebenssituationen der Menschen zwischen 30 und 49 Jahren häufig einen Hinderungsgrund für die Aufnahme oder Beibehaltung eines freiwilligen Engagements dar. Das mittlere Lebensalter wird in der Forschung auch häufig als die ,Rushhour des Lebens' bezeichnet. Bedingt durch verschiedene, gleichzeitig auftretende demografische Entwicklungen, sind Menschen im mittleren Lebensalter heutzutage häufiger mit multiplen Problemstellungen konfrontiert als noch in früheren Jahrzehnten (Infurna et al. 2020). So jonglieren heute mehr Menschen im mittleren Lebensalter als früher die Verantwortlichkeiten von Kindererziehung, Pflege von Angehörigen und beruflicher Karriere gleichzeitig. Möglicherweise nennen Menschen im Alter von 30 bis 49 Jahren deshalb tendenziell anteilig häufiger zeitliche, berufliche und familiäre Faktoren als Hinderungs- oder Beendigungsgründe ihres Engagements. 75,o Prozent der zurzeit nicht-engagierten Personen in dieser Altersgruppe sind prinzipiell dazu bereit, sich zu engagieren. Eine zeitliche Flexibilisierung und Erleichterungen für die zeitliche Reduzierung von Engagementaufgaben seitens der Organisationen und Vereine, indem beispielsweise Verantwortlichkeiten geteilt werden, könnten es mehr Menschen im Alter von 30 bis 49 Jahren erlauben, trotz ihrer hohen zeitlichen Einbindung freiwillig tätig zu sein. 
Im Zuge des demografischen Wandels steht die Beteiligung älterer Menschen am freiwilligen Engagement immer stärker im Fokus zivilgesellschaftlicher Initiativen (Burkhard \& Schupp 2019; Erlinghagen 2008; Kubisch \& Störkle 2016). Betont wird in diesem Zusammenhang auch die Bedeutung, die das Engagement haben kann, um die durch den Übergang in den Ruhestand wegfallenden Möglichkeiten zu sozialen Interaktionen zu kompensieren. Ein Beispiel hierfür ist das Bundesprogramm „Mehrgenerationenhaus“ (BMFSFJ 2020a), welches genau darauf abzielt verschiedene Generationen zusammenzubringen. Trotz des möglichen Nutzens des Engagements sowohl für die älteren Menschen selbst als auch für die Gesellschaft, darf man nicht vergessen, dass es ,die älteren Menschen' als homogene Gruppe nicht gibt und sich hinter dieser Altersgruppe eine große heterogene Gruppe mit unterschiedlichen Fähigkeiten und Motiven verbirgt (Motel-Klingebiel et al. 2010). Es gibt neben der Gruppe älterer Menschen, die sowohl die nötige körperliche Fitness als auch die Motivation für ein Engagement mitbringen, auch einen großen Anteil derjenigen, die entweder körperlich, gesundheitlich oder durch innerfamiliäre Übernahme von Pflege- und Betreuungsaufgaben zu sehr eingeschränkt sind oder auch ihre Freizeit anders verbringen möchten. Außerdem lässt sich feststellen, dass die prinzipielle Bereitschaft für ein Engagement bei den Nicht-Engagierten, die 65 Jahre oder älter sind, mit 27,2 Prozent vergleichsweise gering ist. Um älteren Menschen den Zugang zum Engagement zu erleichtern, könnten zum Beispiel bei gesundheitlich eingeschränkten Menschen digitale Engagementformen gefördert werden. Dabei ist jedoch zu beachten, dass älteren Menschen häufig sowohl der Zugang zum Internet als auch die Erfahrung mit digitalen Medien fehlt (siehe Kapitel 11; Deutscher Bundestag 2020). Das bedeutet, dass Angebote für digitales Engagement für Ältere von entsprechenden Schulungen begleitet werden müssten. Bei der Werbung älterer Personen für das Engagement müssen auch die sozialen Möglichkeiten einer freiwilligen Tätigkeit noch stärker als bisher betont werden. Da für ältere Engagierte auch die Reputation und der Einfluss, die sie durch das Engagement gewinnen, ein wichtiges Motiv für ein freiwilliges Engagement darstellen, sollte dies bei der Gewinnung älterer Engagierter nicht vernachlässigt werden. Dazu kann es unter Umständen angeraten sein, negative Altersstereotype, wie zum Beispiel, dass ältere Personen zu langsam, zu unflexibel oder nicht ausreichend belastbar seien, die innerhalb der Organisation vorherrschen könnten, abzubauen und starre Altersgrenzen abzuschaffen. Immerhin beenden deutlich mehr Engagierte aus dieser Altersgruppe - nämlich 41,1 Prozent - ihre freiwillige Tätigkeit, weil sie eine Altersgrenze erreicht haben, als aus gesundheitlichen Gründen - hier sind es 28,8 Prozent.

Insgesamt zeigen die Ergebnisse dieses Kapitels ein positives Bild auf. Die Engagementbereitschaft nicht-engagierter Personen ist hoch und für bereits engagierte Personen spielen altruistische sowie soziale Gründe bei der Ausübung ihres freiwilligen Engagements eine deutlich größere Rolle als eigennützige Motive. Die am häufigsten genannten Gründe für die Beendigung oder Nicht-Aufnahme eines Engagements hängen weniger mit dem Engagement selbst als vielmehr mit äußeren Umständen 
zusammen. Die Zeit ist hier der mit Abstand am häufigsten genannte Grund. Aus beruflichen, familiären oder auch anderen Gründen scheint es für Nicht-Engagierte sehr schwierig, die nötige Zeit zu finden, um ein freiwilliges Engagement in ihr Leben zu integrieren. Eine zeitliche Flexibilisierung des freiwilligen Engagements seitens der Organisationen und Vereine, sowie eine bessere Informationsarbeit, Organisation und Vernetzung von Vereinen und Projekten könnten den Zugang zu freiwilligem Engagement für Interessierte deutlich erleichtern und so zu mehr und dauerhafterem Engagement führen.

\section{Literatur}

Bundesanstalt Technisches Hilfswerk (THW) (Hrsg.) (2020). Deine Zeit ist jetzt. Online: https://jetzt.thw.de/DE/Startseite/startseite.html (zuletzt abgerufen 19.11. 2020).

Bundesministerium für Familie, Senioren, Frauen und Jugend (BMFSFJ) (Hrsg.) (2020a). Mehrgenerationenhaus. Miteinander - Füreinander. Online: https://www.mehrgenerationenhaeuser.de/ (zuletzt abgerufen 19.11.2020).

Bundesministerium für Familie, Senioren, Frauen und Jugend (BMFSFJ) (Hrsg.) (202ob). Menschen stärken Menschen. Online: https:/www.bmfsfj.de/bmfsfj/themen/engagementund-gesellschaft/engagement-staerken/menschen-staerken-menschen (zuletzt abgerufen 19.11.2020).

Burkhardt, L., \& Schupp, J. (2019). Wachsendes ehrenamtliches Engagement: Generation der 68er häufiger auch nach dem Renteneintritt aktiv. DIW Wochenbericht Nr. 42. Online: https:// www.diw.de/documents/publikationen/73/diw_01.c.683542.de/19-42-1.pdf (zuletzt abgerufen: 10.09. 2020).

Deutscher Bundestag (2020). Achter Bericht zur Lage der älteren Generation in der Bundesrepublik Deutschland. Ältere Menschen und Digitalisierung und Stellungnahme der Bundesregierung (Drucksache 19/21650). Berlin: Deutscher Bundestag.

Engagierte Stadt (Hrsg.) (2020). Willkommen in der Engagierten Stadt. Online: https://www. engagiertestadt.de/ (zuletzt abgerufen 19.11.2020).

Erlinghagen, M. (2008). Informelle Arbeit in alternden Gesellschaften. Möglichkeiten und Grenzen des „produktiven Alterns“. Schmollers Jahrbuch, 128(2), 237-259.

Haumann, W. (2014). Motive des bürgerschaftlichen Engagements. Kernergebnisse einer bevölkerungsrepräsentativen Befragung durch das Institut für Demoskopie Allensbach im August 2013. Berlin: Bundesministerium für Familie, Senioren, Frauen und Jugend (BMFSFJ).

Hessische Landesregierung (Hrsg.) (2020). \#deinehrenamt. Online: https://www.deinehrenamt.de/Start (zuletzt abgerufen 29.04.2021).

Huxhold, O., \& Hameister, N. (2017). Soziale Einbettung und freiwilliges Engagement. In: J. Simonson, C. Vogel \& C. Tesch-Römer (Hrsg.) Freiwilliges Engagement in Deutschland - Der Deutsche Freiwilligensurvey 2014 (S. 499-520). Wiesbaden. Springer VS.

Infurna, F. J., Gerstorf, D., \& Lachman, M.E. (2020). Midlife in the 2020s: Opportunities and challenges. American Psychologist, 75(4), 470-485. 
Institut für Demoskopie Allensbach (2013). Motive des bürgerschaftlichen Engagements. Ergebnisse einer bevölkerungsrepräsentativen Befragung. Untersuchung im Auftrag des Bundesministeriums für Familie, Senioren, Frauen und Jugend (BMFSFJ). Allensbach.

Klaus, D., \& Vogel, C. (2019). Unbezahlte Sorgetätigkeiten von Frauen und Männern im Verlauf der zweiten Lebenshälfte. In: C. Vogel, M. Wettstein \& C. Tesch-Römer (Hrsg.) Frauen und Männer in der zweiten Lebenshälfte: Älterwerden im sozialen Wandel (S. 91-112). Wiesbaden: Springer VS.

Kubisch S. \& Störkle M. (2016). Erfahrungswissen in der Zivilgesellschaft. Eine rekonstruktive Studie zum nachberuflichen Engagement. Wiesbaden: Springer VS.

Motel-Klingebiel, A., Wurm, S., Huxhold, O., \& Tesch-Römer, C. (2010). Wandel von Lebensqualität und Ungleichheit in der zweiten Lebenshälfte. In: A. Motel-Klingebiel, S. Wurm, O. Huxhold \& C. Tesch-Römer (Hrsg.) Altern im Wandel. Befunde des Deutschen Alterssurveys (DEAS) (S. 15-33). Stuttgart: Kohlhammer.

Moschner, B. (2002). Altruismus und Egoismus. Was motiviert zum Ehrenamt? In: Zeitschrift für Politische Psychologie, 10 (1-2), 25-40.

Müller, D., Hameister, N., \& Lux, K. (2017). Anstoß und Motive für das freiwillige Engagement. In: J. Simonson, C. Vogel \& C. Tesch-Römer (Hrsg.) Freiwilliges Engagement in Deutschland Der Deutsche Freiwilligensurvey 2014 (S. 413-435). Wiesbaden: Springer VS.

Müller, D., \& Tesch-Römer, C. (2017). Früheres Engagement und Engagementbereitschaft. In: J. Simonson, C. Vogel \& C. Tesch-Römer (Hrsg.) Freiwilliges Engagement in Deutschland - Der Deutsche Freiwilligensurvey 2014 (S. 153-178). Wiesbaden: Springer VS.

Seebauer, S. (2013). Bereitschaft und Motive für Freiwilligenarbeit, VOICE Working Paper o1. Graz: Wegener Center für Klima und Globalen Wandel, Karl-Franzens-Universität Graz.

Shye, S. (2009). The Motivation to Volunteer: A Systemic Quality of Life Theory. Social Indicators Research, 98(2), 183-200.

Wagner, G. (2019). Helfen und Reziprozität. Freiwilliges Engagement für Geflüchtete im ländlichen Raum. Zeitschrift für Soziologie, 48(3), 226-241.

Wenzel, D., Beerlage, I., \& Springer, S. (2012). Attraktivität und Beendigung von freiwilligem Engagement. In: D. Wenzel, I. Beerlage \& S. Springer (Hrsg.) Motivation und Haltekraft im Ehrenamt. Die Bedeutung von Organisationsmerkmalen für Engagement, Wohlbefinden und Verbleib in Freiwilliger Feuerwehr und THW (S. 54-74). Herbolzheim: Centaurus Verlag \& Media. 
Open Access Dieses Kapitel wird unter der Creative Commons Namensnennung Nicht kommerziell 4.0 International Lizenz (http://creativecommons.org/licenses/bync/4.0/deed.de) veröffentlicht, welche die nicht-kommerzielle Nutzung, Vervielfältigung, Bearbeitung, Verbreitung und Wiedergabe in jeglichem Medium und Format erlaubt, sofern Sie den/die ursprünglichen Autor(en) und die Quelle ordnungsgemäß nennen, einen Link zur Creative Commons Lizenz beifügen und angeben, ob Änderungen vorgenommen wurden.

Die in diesem Kapitel enthaltenen Bilder und sonstiges Drittmaterial unterliegen ebenfalls der genannten Creative Commons Lizenz, sofern sich aus der Abbildungslegende nichts anderes ergibt. Sofern das betreffende Material nicht unter der genannten Creative Commons Lizenz steht und die betreffende Handlung nicht nach gesetzlichen Vorschriften erlaubt ist, ist auch für die oben aufgeführten nicht-kommerziellen Weiterverwendungen des Materials die Einwilligung des jeweiligen Rechteinhabers einzuholen. 